\title{
Élaboration d'un système de surveillance des cellules, des tissus et des
}

\section{organes}

\author{
Hong $Z^{1 *}$, Kenny $P^{1}$, Hyson $C^{1}$, Archibald $C^{1}$ \\ ${ }^{1}$ Centre de la lutte contre les maladies transmissibles et les infections, Agence de la santé publique du Canada, Ottawa (Ontario) \\ *Correspondance: zhiyong.hong@phac-aspc.gc.ca
}

\section{Résumé}

Contexte : Un nombre croissant de transplantations de cellules, de tissus et d'organes sont effectuées chaque année dans les établissements de soins de santé du Canada, y compris les cliniques et les cabinets de médecin et de dentiste. L'Agence de la santé publique du Canada (I'Agence) dirige un projet d'élaboration d'un système de surveillance des cellules, des tissus et des organes (SSCTO).

Objectif : Créer des données opportunes, utiles et pertinentes à l'échelle nationale sur les événements indésirables liés aux transplantations en appuyant l'élaboration ou le renforcement des systèmes de collecte de données des provinces et territoires.

Méthodologie : Des éléments de données minimaux et des définitions ont été établis pour les tissus en se référant aux définitions établies par l'Union européenne et les États-Unis. La collecte de données sur les événements indésirables liés aux allogreffes de tissus humains a démarré en avril 2011 dans des sites pilotes, en Alberta, en Ontario, au Québec, au Nouveau-Brunswick et en Nouvelle-Écosse.

Résultats : En décembre 2013, on dénombrait 8 cas déclarés d'événements indésirables liés aux greffes de tissus, dont 7 concernant des greffes de tissu cornéen et 1 concernant une greffe de tissu cardiovasculaire.

Conclusion : Le déploiement à grande échelle d'un SSCTO pourrait augmenter la capacité du Canada à améliorer la sécurité des patients. La collecte et l'analyse des données pourraient permettre de mieux cerner les événements indésirables liés aux transplantations, et par là même éclairer la mise au point de stratégies destinées à prévenir et réduire la gravité de ces événements. La prochaine étape du développement du SSCTO sera d'établir des éléments de données et des définitions pour les volets relatifs à la transplantation de cellules et d'organes du système et d'élargir le nombre de sites pilotes participant à la surveillance.

\section{Introduction}

Contexte : Un nombre croissant de greffes de cellules, de tissus et d'organes sont effectuées chaque année dans les établissements de soins de santé du Canada, y compris les cliniques et les cabinets de médecin et de dentiste (1). Au Canada, plus de 90000 tissus allogreffes sont distribués pour être greffés, y compris les tissus musculo-squelettiques, vasculaires, cutanés, cardiaques et cornéens (2). Selon les prévisions de la Société canadienne du sang, le besoin de transplantations d'organes augmentera considérablement au cours des deux prochaines décennies (3). L'Agence de la santé publique du Canada (I'Agence) dirige l'élaboration d'un Système de surveillance des cellules, des tissus et des organes (SSCTO), dont l'objectif est d'améliorer la sécurité des transplantations pour les Canadiens grâce à la saisie et à l'analyse de données relatives aux événements indésirables et à la diffusion des connaissances ainsi acquises.

L'acquisition de données de surveillance sur les événements indésirables liés aux transplantations est essentielle à la création de programmes et de politiques appropriés qui nous permettront d'améliorer la sécurité des patients. Une fois déployé à grande échelle, le système collectera des données sur les événements indésirables modérés et graves dans le but de surveiller l'évolution des risques connus et des nouveaux risques ainsi que de réduire la transmission des maladies infectieuses due aux transplantations. Le présent article a pour objectif de résumer l'état d'avancement du projet d'élaboration du système de surveillance des événements indésirables liés aux transplantations à trois volets (4). 


\section{Méthodologie}

Des éléments de données minimaux et des définitions d'événements indésirables graves ont été élaborés en se référant aux définitions établies par l'Union européenne et les États-Unis. La classification de la gravité de l'événement indésirable (nulle, mineure, grave, décès potentiel et décès) a été définie et elle est appliquée à la déclaration de cas. Le système de classification pour l'imputabilité a été appliqué pour évaluer la probabilité qu'un événement indésirable chez un receveur puisse être attribué au processus du don ou à l'application clinique des tissus appliqués. La collecte de données sur les événements indésirables liés aux allogreffes de tissus humains a démarré en avril 2011 dans des sites pilotes, en Alberta, en Ontario, au Québec, au Nouveau-Brunswick et en Nouvelle-Écosse. Les méthodes de collecte des déclarations d'événements indésirables liés à la transplantation variaient d'un site pilote à l'autre.

\section{Résultats}

En décembre 2013, on dénombrait 8 cas signalés d'événements indésirables liés à des greffes de tissus, dont la majorité concernait des tissus cornéens (tableau 1). Cinq événements indésirables liés à des greffes de tissus cornéens ont été déclarés à l'Agence par le site pilote de l'Alberta depuis le $1^{\mathrm{er}}$ avril 2011 ; un événement de ce type a été déclaré par le site pilote de la Nouvelle-Écosse en 2011. Un événement indésirable grave lié à des tissus cardiovasculaires et un événement indésirable lié à des tissus cornéens ont été déclarés à l'Agence par le site pilote du Québec respectivement en 2012 et 2013. Dans l'ensemble, la plupart des sites de surveillance ont produit des rapports complets et annuels sur les événements indésirables liés aux greffes. En général, il a fallu de trois à six mois au site et à l'établissement fournisseur de tissus pour mener une enquête complète sur un événement indésirable soupçonné. Plusieurs facteurs ont influé sur la déclaration des événements, y compris : l'expertise et l'expérience du médecin traitant dans la détection des événements chez les receveurs, l'efficacité de la coordination entre les organismes d'approvisionnement et les hôpitaux, ainsi que la charge de travail des coordonnateurs.

Tableau 1 : Aperçu des événements indésirables liés aux greffes de tissus en fonction des sites participant au SSCTO ${ }^{1}$ pour la période allant d'avril 2011 à décembre 2013

\begin{tabular}{|c|c|c|c|c|c|}
\hline Année & Site 1 & Site 2 & Site 3 & Site 4 & Site 5 \\
\hline $\mathbf{2 0 1 1}$ & 0 & 0 & 0 & 0 & 1 \\
\hline $\mathbf{2 0 1 2}$ & 4 & 0 & 1 & 0 & 0 \\
\hline $\mathbf{2 0 1 3}$ & 1 & 0 & 1 & 0 & 0 \\
\hline Total & $\mathbf{5}$ & $\mathbf{0}$ & $\mathbf{2}$ & $\mathbf{0}$ & $\mathbf{1}$ \\
\hline
\end{tabular}

${ }^{1} \mathrm{SSCTO}=$ Système de surveillance des cellules, des tissus et des organes

\section{Conclusion}

Le système de surveillance des cellules, des tissus et des organes du Canada renforce notre capacité à cerner les événements indésirables liés à la transplantation. L'amélioration de la collecte et de l'analyse des données nous permettra de mieux comprendre ces événements et d'acquérir les connaissances nécessaires à l'élaboration de stratégies visant à les prévenir ou à les réduire. La prochaine étape du développement du SSCTO sera d'établir des éléments de données et des définitions pour les volets relatifs aux cellules et aux organes du système et d'élargir le nombre de sites participant à la surveillance.

\section{Remerciements}

L'Agence tient à remercier les établissements de soins de santé suivants pour leur contribution à la surveillance des événements indésirables liés aux transplantations de tissus et d'organes : les services de transplantation de l'organisme Alberta Health Services (Alberta), Héma-Québec et le ministère de la Santé et des Services sociaux du Québec 
(Québec), l'hôpital Sunnybrook, Toronto (Ontario), la Banque d'yeux et de tissus du Nouveau-Brunswick et le bureau provincial de coordination du sang de la Nouvelle-Écosse (Nouvelle-Écosse). De plus, l'Agence a fortement apprécié les suggestions et commentaires émis par la Direction des produits de santé commercialisés, Direction générale des produits de santé et des aliments, Santé Canada, ainsi que la Société canadienne du sang lors de l'élaboration du présent document.

\section{Conflit d'intérêts}

Aucun

\section{Financement}

Le financement de ce projet a été assuré par l'Agence de la santé publique du Canada.

\section{Références}

(4) 